\title{
Propuesta de un nuevo puntaje para optimizar estimados ecográficos de peso fetal: estudio piloto
}

\author{
New score to optimize ultrasound fetal weight estimation: a pilot study
}

\author{
José Rojas Camayo ${ }^{1}$, Miguel Garay ${ }^{1}$, César Ortiz¹, Héctor Flores ${ }^{1}$, Fabiola Huaroto ${ }^{1}$, \\ Hugo Chico1, Iván Huamaní1, Javier Valencia1, Carlos Paz-Soldán \\ ${ }^{1}$ Hospital Nacional Guillermo Almenara Irigoyen, Lima-Perú.
}

\begin{abstract}
Resumen
Introducción: La estimación del peso fetal es importante para las decisiones obstétricas; la precisión de los estimados ecográficos no ha sido estudiada. Objetivos: Determinar la precisión del estimado ecográfico del peso fetal mediante un puntaje basado en parámetros clínicos, ecográficos y maternos. Diseño: Estudio de tipo transversal. Lugar: Hospital Nacional Guillermo Almenara Irigoyen, Lima, Perú. Participantes: 130 gestantes y sus fetos. Intervenciones: Se consignó datos clínicos, ecográficos y maternos. Se procedió a dicotomizar los aciertos entre menor o igual a $\pm 10 \%$ (acertadas) y mayor a $\pm 10 \%$ (erradas) del peso real. Se analizó las variables clínicas, ecográficas y maternas mediante el uso de las curvas ROC, para identificar aquellas que tuvieron más influencia en la precisión ecográfica. Con las variables identificadas, se procedió al desarrollo de un modelo de regresión logística, para establecer un puntaje que estimara la probabilidad de variación, teniendo como punto de corte $\pm 10 \%$ del peso real. Principales medidas de resultados: Precisión de un puntaje que relaciona la edad gestacional con la circunferencia abdominal y el diámetro biparietal del feto, el ponderado clínico y el peso materno. Resultados: El nuevo puntaje tuvo un buena capacidad de discriminación de las ecografías, según su variación a $\pm 10 \%$ del peso real (área bajo la curva ROC: $0,76 p<0,001$ ). Según los puntajes obtenidos, se obtuvo tres grupos: rendimiento alto $20 \%$, rendimiento convencional $44,4 \%$ y rendimiento bajo $71,2 \%$. Conclusiones: El nuevo puntaje propuesto tiene la capacidad de estimar la probabilidad de variación de los estimados ecográficos mayores al $\pm 10 \%$ del peso real, conformando tres grupos de rendimiento: alto, convencional y bajo.

Palabras clave: Ecográfica; peso fetal; curva ROC.
\end{abstract}

\begin{abstract}
Introduction: Fetal weight estimation is important for obstetrical decisions; accuracy of ultrasound fetal weight estimations has not been studied. Objective: To determine ultrasound fetal weight estimations precision by developing a score based on clinical, ultrasound and maternal parameters. Design: Cross sectional study. Setting: Guillermo Almenara Irigoyen National Hospital, Lima, Peru. Participants: 130 pregnant women and their fetuses. Interventions: Clinical, ultrasound and maternal data were collected. We dichotomized predictions as actual birth weight less or equal to $\pm 10 \%$ (correct) and more than $\pm 10 \%$ (erroneous). We analyzed clinical, ultrasonographic and maternal variables using ROC curves in order to identify those with more influence in ultrasound accuracy. With such identified variables we developed a logistic regression model to establish a score that would estimate the probability of variation, having a $\pm 10 \%$ of real fetal birth weight cut point. Main outcome measures: Precision of a score that relates gestational age and both fetal abdominal circumference and biparietal diameter, clinical fetal weight estimation and maternal weight. Results: The resulting score had a good capacity of discrimination of ultrasonographic fetal weight estimations according to their variation to $\pm 10 \%$ of real birth weight (area under the curve ROC: 0,76 $p<0,001$ ). Three groups resulted: high performance $20 \%$, conventional performance $44,4 \%$, and low performance $71,2 \%$. Conclusions: The new score proposed estimates the probability of variation of fetal weight above $\pm 10 \%$ of real birth weight, with three groups of performance: high, conventional and low.
\end{abstract}

Key words: Ultrasonography; fetal weight; ROC curve.

\section{INTRODUCCIÓN}

La estimación del peso fetal mediante ultrasonografía es de mucha utilidad para la toma de decisiones en el manejo obstétrico, por ejemplo, en la detección de macrosomía fetal. Diversos autores han demostrado que el peso incrementado aumenta las posibilidades de traumatismo obstétrico; Boyd y col. mencionan un aumento del riesgo de daño de plexos nerviosos de $0,04 \%$ a $4 \%$ cuando el peso se incrementa de 2500 - $3999 \mathrm{~g}$ a más de $4000 \mathrm{~g}{ }^{(1)}$; Wikström y col. observaron $8 \%$ de traumatismo obstétrico en neonatos mayores de $4500 \mathrm{~g}$ comparados con 0,6\% en los de peso normal ${ }^{(2)}$; Kolderup y col. encontraron un riesgo relativo de 6,7 (IC95\%: 6,5 a 6,9) de injuria obstétrica en neonatos con pesos mayores de $4000 \mathrm{~g}$, comparados con neonatos entre 3000 y $3999 \mathrm{~g}^{(3)}$.
Numerosas fórmulas para el cálculo del peso fetal ecográfico han sido desarrolladas con diferentes grados de exactitud; sin embargo, ninguna de ellas es consistentemente superior ${ }^{(4)}$. Se postula que muchos factores pueden influenciar en la exactitud de la estimación ecográfica del peso fetal; una de ellas es la experiencia del ecografista, como lo demuestra un estudio realizado en médicos residentes, en quienes los de menor de experiencia (menos de 6 meses) 49,4\% de sus estimados ecográficos estuvieron dentro del 10\% del peso real, mientras que en los más experimentados (más de 24 meses) fue de $73,6 \%{ }^{(5)}$. Sin embargo, aún entre ecografistas hay diferencias, como lo muestra un estudio donde tres ecografistas experimentados realizaron ecografías de forma independiente a 39 gestantes a término y hubo diferencias en mayor grado en las medidas de la circunferencia abdominal y circunferencia cefálica que en las medidas del diámetro biparietal y longitud de fémur; además, la discrepancias del 10\% del peso real al nacimiento fueron menores (se redujo aproximadamente 50\%), cuando se tomó en cuenta la estimación de los tres ecografistas experimentados (6). Uno de los parámetros que más está sometido a variación es la medición de la circunferencia abdominal ${ }^{(6,7)}$. Otros factores, que aún son controversiales, también pueden influenciar la exactitud ecográfica: la etnicidad, índice de masa corporal materno, sexo fetal, multiparidad y volumen de líquido amniótico ${ }^{(4)}$.

Tal como hemos señalado, aún no se ha desarrollado un instrumento que ayude a identificar el grado de exactitud 
de un estimado ecográfico, que podría ser de gran utilidad clínica en la toma de decisiones obstétricas. El propósito del presente estudio fue desarrollar un puntaje basado en parámetros clínicos, ecográficos y maternos mediante el uso de las curvas ROC (receiver operating characteristics) y de la estadística multivariante, para poder estimar la probabilidad de variación mayor de $\pm 10 \%$ entre el peso ecográfico estimado y el peso real al nacimiento.

\section{MÉTODOS}

Se realizó un estudio observacional, transversal, en el que se recolectó datos de forma prospectiva de 130 nacimientos, de 30 a 42 semanas, durante los meses de octubre a diciembre del año 2007, en el Servicio de Obstetricia del Hospital Nacional Guillermo Almenara, Lima, Perú. Se obtuvo los estimados ecográficos como máximo hasta siete días antes del nacimiento; no se incluyó embarazos gemelares ni malformaciones congénitas mayores. Todas las ecografías consideradas para el estudio fueron realizadas en el hospital mediante dos ecógrafos, ambos sistemas de ultrasonido GE logiq 400 MD (General Electric Medical Systems Milwaukee, Wis), con transductor curvilíneo de 3,5 MHz. Ambos aparatos utilizaron la fórmula de Hadlock $2^{(8)}$ para el cálculo estimado del peso fetal, donde se evaluó los parámetros de diámetro biparietal (DBP), circunferencia abdominal (CA) y longitud de fémur (LF). Las ecografías fueron realizadas por 15 médicos ginecoobstetras, de los cuales 4 hicieron entre 15 y 33 ecografías; 7 médicos entre 5 y 8 ecografías; y 4 médicos menos de 5 ecografías cada uno, los cuales no tuvieron conocimiento del estudio para así evitar el sesgo de que intentaran mejorar sus habilidades y por ende los resultados no serían como en la practica clínica cotidiana.

Para la recolección de datos, se elaboró una ficha, en la que se consignó los siguientes datos maternos: edad gestacional (EG), paridad, peso materno, talla materna, fecha de última regla, edad gestacional por fecha de última regla o ecografía del primer trimestre, altura uterina, altura de presentación, fecha de medida de altura uterina. Los datos ecográficos fueron: fecha y hora de ecografía, profesional que realizó la ecografía, sexo del feto, diámetro biparietal, circunferencia cefálica, circunferencia abdominal, longitud del fémur, ponderado fetal ecográfico e índice de líquido amniótico. Y los datos del recién nacido, fecha de nacimiento, vía de culminación de la gestación, intervalo en días entre la última medición de la altura uterina y el nacimiento, intervalo en días entre la última ecografía y el nacimiento, sexo y peso cuantificado con balanza calibrada.

Para el cálculo del ponderado fetal clínico se utilizó el método de Johnson y Toshach ${ }^{(9)}$, que emplea la altura uterina; para la medición de la misma, se colocó el extremo inferior de la cinta métrica con la mano derecha sobre el borde superior del pubis y el extremo inferior con la mano izquierda, con la cinta colocada entre los dedos índice y medio, limitando perpendicularmente al extremo superior del útero. A estos valores se les restó 12, en las presentaciones por encima de la espina ciática, y 11 a las presentaciones al nivel o por debajo de las espinas ciáticas. Esta diferencia fue multiplicada por 155 y el valor fue anotado como estimado clínico del peso fetal. Los datos mencionados fueron recolectados por siete internos de medicina en su última rotación de internado, de forma prospectiva (durante prácticas de neonatología), luego de haber rotado por ginecoobstetricia; de esta manera se aseguró el buen conocimiento de los datos obstétricos. Se obtuvo los datos de la historia clínica, sobre todo los datos obstétricos.y ecográficos, considerándose para el estudio la última ecografía realizada en el Hospital Nacional Guillermo Almenara Irigoyen; y, si algún dato faltaba, se entrevistó a la paciente personalmente (el estimado ecográfico no debía ser mayor a 7 días antes del parto).

Para el análisis, se creó una base de datos en el programa estadístico SPSS v. 15. Primeramente, para nuestra variable principal se halló la diferencia entre el peso al nacimiento y el peso estimado ecográfico. Y se consideró como punto de corte para evaluar la precisión una variación mayor de $\pm 10 \%$ del peso real. De esta forma se obtuvo dos grupos: uno de variación mayor de $\pm 10 \%$ del peso real (erradas) y el otro grupo de variación hasta $\pm 10 \%$ del peso real (acertadas). Se consiguió así una variable dicotómica, la cual pasó a ser la variable dependiente para los análisis respectivos.

Para la identificación de las variables que pudieran influir en la probabilidad de mayor o menor variación de $\pm 10 \%$ del peso real, se realizó una serie de análisis usando las curvas ROC. De este modo, se analizó cada una de las variables mencionadas anteriormente y se seleccionó las que tuvieran mejor área bajo la curva, que nos identificaran cuáles eran las que mejor influían si una ecografía tenía más probabilidad en el resultado de una variación mayor de $\pm 10 \%$ del peso real.

Una vez seleccionadas las variables, se procedió a identificar el mejor punto de corte, mediante la visualización de la curva ROC y se buscó el punto más alejado si se trazaba una línea paralela a la diagonal de referencia. Una vez identificados los mejores puntos de corte de las variables de mayor influencia (en dos rangos), se procedió a realizar un modelo de regresión logística con dichas variables, para identificar sus odd ratios ajustados, que nos estimaran la influencia que tenían en la probabilidad de variación mayor de $\pm 10 \%$ del peso real. Estos odd ratios fueron tomados como puntos de referencia, para el puntaje, y subdividiéndose después en tres grupos según los puntajes obtenidos.

En cada subgrupo (definido por la puntuación) se realizó primeramente un análisis de estadística descriptiva, al mostrar porcentaje de variación mayor o menor a $\pm 10 \%$ del peso real (porcentaje de errados). Luego, a cada subgrupo: a) se calculó la media de error absoluto en gramos y en porcentaje, con sus respectivas desviaciones estándar; b) seguidamente, el promedio de la diferencia entre el peso al nacimiento (PAN) y el peso estimado ecográficamente (PEE); además, se mostró su desviación estándar; c) se calculó el porcentaje de sesgo de error, que se obtuvo de la siguiente manera: \% sesgo de error $=100 \mathrm{x}(\mathrm{PAN}-\mathrm{PEE}) / \mathrm{PEE}$; de la misma manera, se obtuvo una desviación 
estándar del porcentaje sesgo de error; d) posteriormente, los coeficientes de correlación intraclase por cada rango mencionado.

\section{RESULTADOS}

Para el análisis del puntaje, 130 ecografías contaban con los datos necesarios; de ellas, 64 (49,2\%) correspondieron a pacientes primíparas. La culminación del embarazo fue por cesárea en 86 (66,2\%) casos y parto vaginal en $44(33,8 \%)$. Los neonatos fueron 78 (60\%) masculinos y $52(40 \%)$ femeninos. En la tabla 1, podemos visualizar la edad materna promedio de 31,5 años, edad gestacional desde 30 a 42 semanas, estimados clínicos fetales desde $1550 \mathrm{~g}$ a $4185 \mathrm{~g}$, estimados ecográficos entre 1364 y 4115 g y pesos neonatales de 1290 a $4560 \mathrm{~g}$.

En la tabla 2 observamos que, entre los diferentes médicos que realizaron más de 10 ecografías y los que realizaron menos de 10 (presentados en un solo grupo), no existió diferencias significativas en su performance, al estimar los pesos fetales ecográficamente comparados con el peso al nacimiento ( $\left.\mathrm{X}^{2}: 1,199 \mathrm{p}: 0,878\right)$.

Para identificar los parámetros usados en el puntaje, se realizó análisis sucesivos con las curvas ROC a las distintas variables e incluso combinaciones de ellas (tabla 3), lográndose identificar cuatro parámetros (sombreadas) que mejor se ajustaron para predecir la posibilidad de que un estimado de peso ecográfico tuviera una variación mayor al \pm 10 del peso real.

Una vez identificados los cuatro parámetros, se procedió a analizarlos en un modelo de regresión logística, teniendo como variable dependiente dicotómica la variación al $\pm 10 \%$ del peso real, obteniéndose sus odd ratios ajustados, los que fueron tomados como puntos de referencia para asignar los puntajes respectivos (tabla 4).

Con los valores para los puntajes respectivos, se hizo un nuevo análisis para visualizar la curva ROC y el área bajo la curva (figura 1) del nuevo puntaje propuesto. Se obtuvo un área de 0,763 $(p<0,001)$, el cual nos señala que el
Tabla 1. Características generales de los pacientes $(n=130)$.

\begin{tabular}{lrrrr}
\hline & Media & Desviación típica & Mínimo & Máximo \\
\hline Edad materna (años) & 31,5 & 6,1 & 17 & 45 \\
Edad gestacional (semanas) & 37,4 & 3,0 & 30 & 42 \\
Peso materno (kg) & 71,1 & 10,7 & 47 & 107 \\
Talla materna (cm) & 156,5 & 7,0 & 142 & 176 \\
Estimado clínico del peso fetal (g) & 3311,6 & 486,8 & 1550 & 4185 \\
Peso fetal estimado ecográficamente (g) & 2864,3 & 611,9 & 1364 & 4115 \\
Circunferencia abdominal (mm) & 322,6 & 37,4 & 226 & 524 \\
Diámetro biparietal (mm) & 89,9 & 5,0 & 73 & 116 \\
Circunferencia cefálica (mm) & 318,7 & 35,1 & 110 & 514 \\
Longitud del fémur (mm) & 67,9 & 6,6 & 32 & 78 \\
Peso del recién nacido (g) & 3188,7 & 674,4 & 1290 & 4560 \\
Intervalo entre ecografía y nacimiento (días) & 1,95 & 1,8 & 0 & 7 \\
\hline
\end{tabular}

Tabla 2. Performance de los médicos ecografistas al estimar el peso fetal.

\begin{tabular}{|c|c|c|c|c|c|c|c|}
\hline & $\begin{array}{c}\text { Médico } \\
1\end{array}$ & $\begin{array}{l}\text { Médico } \\
2\end{array}$ & $\begin{array}{c}\text { Médico } \\
3\end{array}$ & $\begin{array}{l}\text { Médico } \\
4\end{array}$ & $\begin{array}{l}\text { Médicos } \\
\text { restantes* }\end{array}$ & $X^{2}$ & $p$ \\
\hline Variación $<10 \% \mathrm{n}(\%)$ & $7(53,8)$ & $8(47,1)$ & $12(57,1)$ & $11(42,3)$ & $27(50,9)$ & 1,199 & 0,878 \\
\hline Variación $>10 \% n(\%)$ & $6(46,2)$ & $9(52,9)$ & $9(42,9)$ & $15(57,7)$ & $26(49,1)$ & & \\
\hline Total & 13 & 17 & 21 & 26 & 53 & & \\
\hline
\end{tabular}

* Médicos que realizaron menos de 10 estimados ecográficos cada uno. puntaje tiene buena capacidad de discriminación para identificar ecografías con variación mayor del $\pm 10 \%$ del peso real.

Se subdividió los puntajes en tres subgrupos, para una mejor visualización: a) rendimiento alto (menos de 6 puntos); b) rendimiento convencional (de 6 a 7,9 puntos); y, c) rendimiento bajo (de 8 a 11 puntos). Así obtuvimos los siguientes porcentajes de variación mayor al 10\%: $20 \%$, 44,4\% y $71,2 \%$, respectivamente. Lo cual nos muestra que cada subgrupo
Tabla 3. Área bajo la curva $(\mathrm{ROC})$ de las diferentes variables analizadas, para predecir una variación ecográfica mayor a $\pm 10 \%$ del peso real.

\begin{tabular}{lccccc}
\hline \multicolumn{1}{c}{$\begin{array}{c}\text { Variables resultado } \\
\text { de contraste }\end{array}$} & Área & $\begin{array}{c}\text { Error } \\
\text { típ.(a) }\end{array}$ & $\begin{array}{c}\text { Sig. } \\
\text { asintótica(b) }\end{array}$ & $\begin{array}{c}\text { Intervalo de confianza } \\
\text { asintótico al 95\% } \\
\text { Límite superior }\end{array}$ \\
\hline Edímite inferior \\
Edad materna (años) & 0,49 & 0,07 & 0,85 & 0,35 & 0,62 \\
Peso materno (kg) & 0,64 & 0,07 & 0,05 & 0,50 & 0,77 \\
Índice de masa corporal & 0,56 & 0,07 & 0,40 & 0,42 & 0,69 \\
Estimado clínico del peso fetal & 0,54 & 0,07 & 0,55 & 0,41 & 0,68 \\
Diámetro biparietal (mm) & 0,44 & 0,07 & 0,41 & 0,31 & 0,58 \\
Circunferencia cefálica (mm) & 0,50 & 0,07 & 0,77 & 0,34 & 0,62 \\
Circunferencia abdominal (mm) & 0,29 & 0,06 & 0,98 & 0,37 & 0,64 \\
Longitud del fémur (mm) & 0,50 & 0,07 & 0,963 & 0,17 & 0,42 \\
Índice de líquido amniótico & 0,51 & 0,07 & 0,86 & 0,37 & 0,64 \\
EG x 10 menos CA & 0,81 & 0,05 & 0,000 & 0,38 & 0,65 \\
EG x 10 menos DPB & 0,65 & 0,07 & 0,04 & 0,51 & 0,91 \\
Diferencia clínico ecográfica & 0,63 & 0,07 & 0,07 & 0,49 & 0,78 \\
Peso materno en dos rangos: & & & & & 0,76 \\
$<65$ y de 65 a más & 0,59 & 0,07 & 0,23 & 0,45 & 0,72 \\
\hline
\end{tabular}

(a) Bajo el supuesto no paramétrico.

(b) Hipótesis nula: área verdadera $=0,5$ 
Tabla 4. Modelo de regresión logística.

\begin{tabular}{|c|c|c|}
\hline Variable & $\mathrm{OR}^{\mathrm{a}}$ ajustado & I.C. $95 \%$ \\
\hline \multicolumn{3}{|c|}{ Edad gestacional x 10 - circunferencia abdominal (mm) } \\
\hline Hasta 60 & 1 & \\
\hline De 61 a más & 3,81 & $1,65-8,79$ \\
\hline \multicolumn{3}{|c|}{ Edad gestacional x 10 - diámetro biparietal (mm) } \\
\hline Hasta 290 & 1 & \\
\hline De 291 a más & 2,01 & $0,89-4,54$ \\
\hline \multicolumn{3}{|c|}{ Diferencia entre el estimado clínico y el ecográfico } \\
\hline Hasta 490 & 1 & \\
\hline De 490 a más & 2,52 & $1,12-5,69$ \\
\hline \multicolumn{3}{|l|}{ Peso materno } \\
\hline Menor de $65 \mathrm{~kg}$ & 1 & \\
\hline De $65 \mathrm{~kg}$ a más & 2,83 & $1,17-6,86$ \\
\hline
\end{tabular}

tiene una probabilidad de variación diferente $\left(X^{2}=23,638 p<0,001\right)$, con un porcentaje mayor de variaciones mayor al $\pm 10 \%$ en el subgrupo de rendimiento bajo (figura 2).

En la tabla 5 podemos visualizar distintos parámetros comparados con el total de las ecografías en el estudio, los grupos según el puntaje propuesto (alto, convencional y bajo) y todos ellos comparados con la fórmula Hadlock, en estudios realizados por Nahum y col. ${ }^{(10)}$,
Anderson y col. ${ }^{(11)}$ y Chien y col. ${ }^{(12)}$.

Finalmente, podemos visualizar el puntaje propuesto (tabla 6), con sus puntuaciones respectivas, según los parámetros desarrollados, y los subgrupos según puntajes rendimientos alto, convencional y bajo.

\section{DISCUSIÓN}

El nuevo puntaje propuesto es una herramienta práctica que se basa en

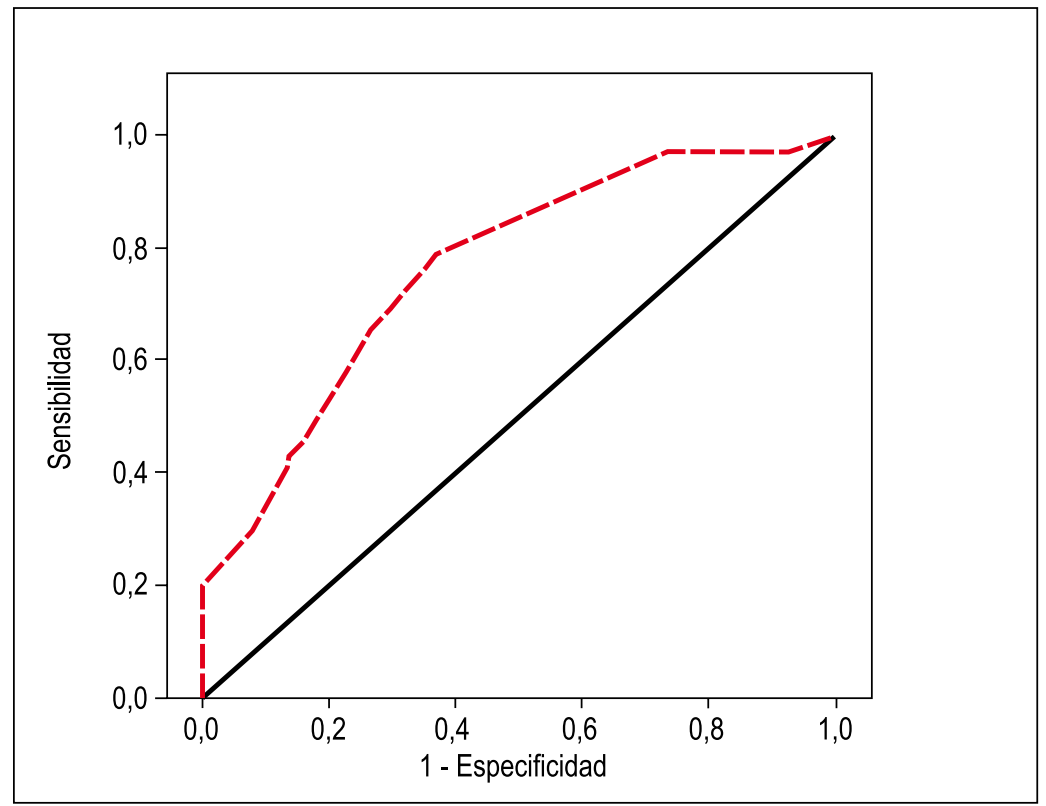

Figura 1. Curva ROC del puntaje para predecir variación ecográfica mayor de $\pm 10 \%$ del peso real. Área bajo la curva: 0,763 $p<0,001$. parámetros objetivos y sencillos -clínicos, ecográficos y maternos-, para poder estimar las probabilidades de variación (mayor al $\pm 10 \%$ de peso real) de los estimados de peso ecográficos. Es además operador independiente que puede servir de parámetro de ayuda para estimar la precisión de una ecografía, ayudando a una mejor selección de las ecografías útiles con estimados más precisos (grupo de rendimiento alto) e identificar los estimados ecográficos con alta probabilidad de variación mayor al $\pm 10 \%$ (grupo de rendimiento bajo). De esta manera puede convertirse en un instrumento de ayuda en las decisiones realizadas por los médicos ginecoobstetras.

Este puntaje es una aproximación distinta a la que usualmente se realiza para mejorar los estimados del peso fetal, donde se ha desarrollado diferentes fórmulas con diferentes grados de precisión ${ }^{(4,11,13)}$. A diferencia de los demás estudios, no se pretendió crear una nueva fórmula para estimar el peso fetal. Según la literatura, se menciona que aún falta mejorar la precisión de las diferentes fórmulas para estimar el peso fetal ${ }^{(4,11)}$. Más bien, se ha buscado en el presente estudio optimizar los resultados de los estimados ecográficos al seleccionar las ecografías de rendimiento alto según los puntajes establecidos y esta manera seleccionar los estimados ecográficos más exactos y mejorar así la precisión ecográfica del estimado del peso fetal; en el caso del estudio se utilizó la formula de Hadlock (que utiliza circunferencia abdominal, longitud de fémur y diámetro biparietal).

Comparando algunos resultados, según los subgrupos identificados por el puntaje propuesto (tabla 5), la media del sesgo de error (\%) del total de las ecografías resulta ser 11,84 . Sin embargo, si se selecciona las ecografías de rendimiento alto según el puntaje, obtenemos una media de sesgo de error mejorada de 3,05\%. Ahora, en cuanto a una desviación estándar de la media del sesgo de error, un parámetro utilizado para estimar la precisión ${ }^{(11)}$, podemos observar que una desviación estándar de los 130 estimados del estudio es de $13,26 \%$, mientras que el grupo de rendimiento alto es de 7,94\%, mostrándonos que se ha optimizado la precisión. 


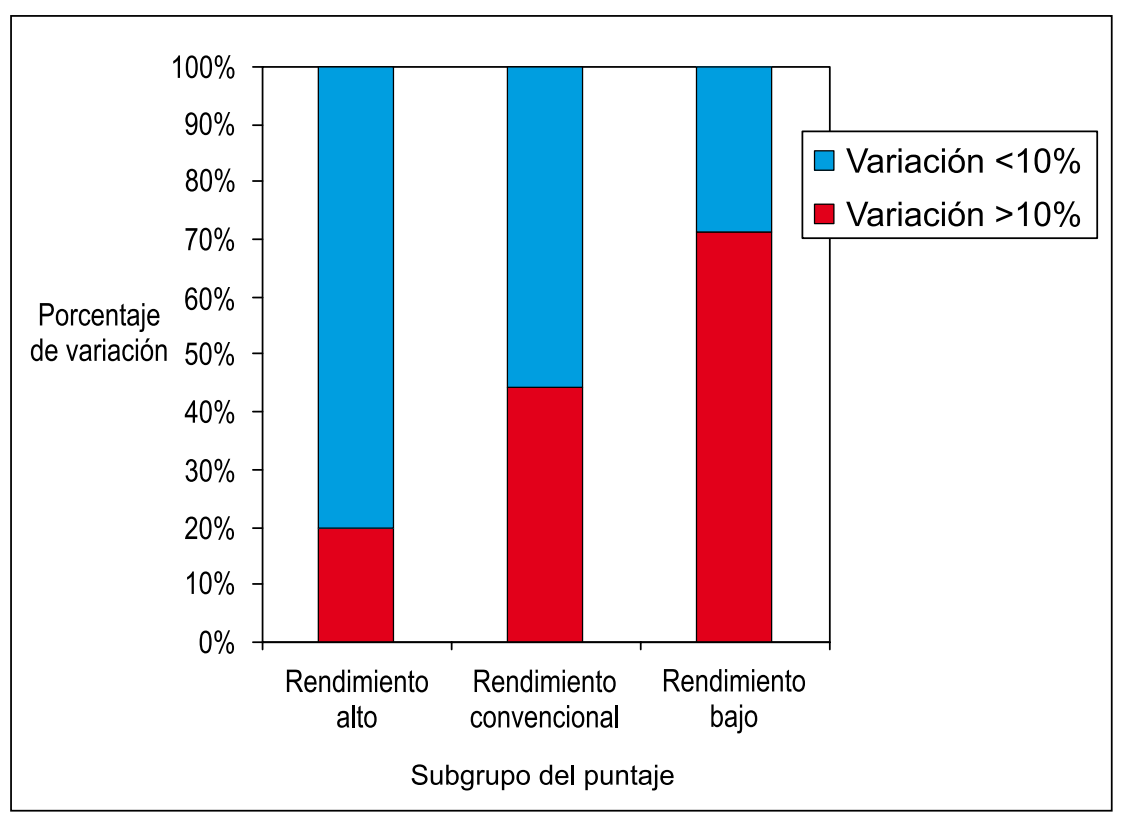

Figura 2. Variación del estimado ecográfico al 10\% del peso real al nacimiento.

Se comparó los resultados con algunos estudios. Un parámetro de comparación es la predicción del peso fetal entre el $10 \%$ del peso real. En nuestro estudio, 65 (50\%) de los 130 estimados ecográficos acertaron entre el $\pm 10 \%$; mientras que en otros estudios, como uno realizado por Nahum y col. ${ }^{(10)}$ encontraron con la fórmula de Hadlock (CA, DBP y LF) un $72 \%$ de aciertos entre el $\pm 10 \%$. Por otro lado, en un estudio realizado por Mirghami y col. ${ }^{(14)}$ en varios grupos étnicos encontraron una predicción de alrededor de $70 \%$ entre el $\pm 10 \%$ para las fórmulas de Shepard (DBP, CA), Warsof (DBP, CA), Hadlock (DBP, CA, CC, LF) y Hadlock (CC, CA, LF). Sin embargo, cuando se realiza el análisis según los subgrupos identificados por el nuevo puntaje propuesto, en el grupo de rendimiento alto encontramos $80 \%$ de aciertos, siendo un porcentaje superior a los comunicados anteriormente; de esta forma se puede visualizar cómo las ecografías son optimizadas para el estimado del peso fetal a través de su clasificación según el puntaje propuesto.

Una de las limitaciones del estudio es haber sido desarrollado en una población mayoritariamente hispana y tomando un solo centro, en el caso de nuestro estudio el Hospital Nacional Guillermo Almenara. Sería interesante realizar estudios multicéntricos y con diferentes grupos étnicos para ampliar el alcance del puntaje; asimismo, utilizar otras fórmulas para el estimado del peso fetal y evaluar la validez de la clasificación usando otras diferentes a la de Hadlock. Otra limitación potencial es que no se realizó cálculos para tomar en cuenta los gramos que aumenta el feto por día, teniendo así un peso ecográfico fetal corregido para cada día, como lo han hecho en dos estudios, uno realizado por Gerard y col. ${ }^{(10)}$, al sumarle $12,4 \mathrm{~g}$ por día a los fetos femeninos y 13,0 g por día si eran masculinos. Y en el estudio realizado por Chien y col. ${ }^{(12)}$, donde se adicionó $25 \mathrm{~g}$ por día, sin diferencia de sexo, a todos los fetos, hasta el día de su nacimiento. Con esta corrección del peso se hubiese obtenido unos resultados donde los estimados hubiesen resultado más precisos, debido a que en general el peso ecográfico fetal se subestima. Sin embargo, no se tomó esta corrección por motivos de practicidad. debido a que es más fácil tomar el peso que figura en la ecografía antes que estar corrigiendo los gramos por cada día. Así, de este modo solo se tomaría como referencia que el tiempo no fuera mayor de 7 días antes del día del nacimiento.

Para concluir, podemos afirmar que el nuevo puntaje propuesto tiene el potencial de poder estimar la probabilidad de que un estimado ecográfico tenga una variación mayor al 10\% con el peso real, de una forma operador independiente. Teniendo el potencial de ser una herramienta útil para la labor asistencial obstétrica.

\section{AGRADECIMIENTOS}

Al Dr. César Gutiérrez Villafuerte, Departamento de Medicina Preventiva UNMSM, por su valioso apoyo en el análisis estadístico.

Tabla 5. Comparación del rendimiento entre las ecografías estudiadas y los subgrupos derivados del puntaje propuesto, y la fórmula de Hadlock.

\begin{tabular}{|c|c|c|c|c|c|c|c|c|c|}
\hline $\begin{array}{c}\text { Grupo de } \\
\text { comparación } \\
\text { (n) }\end{array}$ & $\begin{array}{c}\text { Media de error } \\
\text { absoluto }(\mathrm{g}) \\
\pm 95 \% \mathrm{IC} *\end{array}$ & SD & $\begin{array}{c}\text { Media de error } \\
\text { absoluto (\%) } \\
\pm 95 \% \mathrm{IC}^{\dagger}\end{array}$ & SD & $\begin{array}{c}\text { Media diferencia } \\
\text { peso real - } \\
\text { ecográfico }(\mathrm{g})\end{array}$ & SD & $\begin{array}{c}\text { Media sesgo } \\
\text { de error }{ }^{\ddagger} \\
(\%)\end{array}$ & SD & $\begin{array}{c}\text { Coeficiente } \\
\text { correlación } \\
\text { intraclase }\end{array}$ \\
\hline Total (130) & $366 \pm 48$ & 273,6 & $11,5 \pm 1,4$ & 8,0 & 311,5 & 335,4 & 11,84 & 13,26 & $0,89(0,85-0,92)$ \\
\hline - Alto (35) & $201 \pm 57$ & 165,4 & $6,3 \pm 1,6$ & 4,6 & 99,2 & 243,3 & 3,05 & 7,94 & $0,92(0,85-0,94)$ \\
\hline - Convencional (36) & $342 \pm 87$ & 258,3 & $10,2 \pm 2,5$ & 7,5 & 314,1 & 292,8 & 11,43 & 11,27 & $0,87(0,76-0,93)$ \\
\hline - Bajo (59) & $479 \pm 74$ & 284,0 & $15,3 \pm 2,1$ & 8,0 & 435,9 & 347,6 & 17,31 & 14,15 & $0,91(0,85-0,94)$ \\
\hline Hadlock & $285 \pm 52^{10}$ & & $8,1 \pm 1,5^{10}$ & & $58,0^{11}$ & $244,0^{11}$ & $3,96^{11}$ & $14,67^{11}$ & $0,84(0,73-0,91)^{12}$ \\
\hline
\end{tabular}

\footnotetext{
* error absoluto $(g)=\mid$ PAN-PEE $\mid$

${ }^{\dagger}$ error absoluto $(\%)=\mid 100 x($ PAN-PEE)/PEE $\mid$

$\ddagger \%$ sesgo de error $=100 \times($ PAN-PEE)/PEE
} 
Tabla 6. Parámetros y puntuación.

\begin{tabular}{lc}
\hline \multicolumn{1}{c}{ Parámetro } & Puntajes* $^{*}$ \\
\hline $\begin{array}{l}\text { Edad gestacional x } 10 \text { - Circunferencia abdominal }(\mathrm{mm}) \\
\text { Hasta } 60\end{array}$ & 1 \\
$\quad$ De 61 a más & 4 \\
Edad gestacional x 10 - Diámetro biparietal $(\mathrm{mm})$ & 1 \\
$\quad$ Hasta 290 & 2 \\
De 291 a más & 1 \\
Diferencia entre el estimado clínico y el ecográfico $(\mathrm{g})$ & 2,5 \\
$\quad$ Hasta 490 & \\
$\quad$ De 491 a más & 1 \\
Peso materno & 2,5 \\
$\quad$ Menor de $65 \mathrm{~kg}$ & \\
$\quad$ De 65 kg a más & \\
Subgrupos (probabilidad de variación $>$ al $10 \%$ del peso real $)$ & Menos de 6 \\
Rendimiento alto $(\approx 20 \%)$ & De 6 a 7,9 puntos \\
Rendimiento convencional $(\approx 45 \%)$ & De 8 a 11 puntos \\
Rendimiento bajo $(\approx 70 \%)$ & \\
\hline
\end{tabular}

* Aplicable sólo a estimados ecográficos de 30 semanas a más, y hasta 7 días antes del parto.

\section{REFERENCIAS BIBLIOGRÁFICAS}

1. Boyd ME, Usher RH, McLean FH. Fetal macrosomia: prediction, risks, proposed management. Obstet Gynecol. 1983;61(6):715-22.

2. Wikstrom I, Axelsson 0, Bergstrom R, Meirik 0 . Traumatic injury in large-for-date infants. Acta Obstet Gynecol Scand. 1988;67(3):259-64.

3. Kolderup LB, Laros RK, Jr., Musci TJ. Incidence of persistent birth injury in macrosomic infants: association with mode of delivery. Am J Obstet Gynecol. 1997;177(1):37-41.

4. Dudley NJ. A systematic review of the ultrasound estimation of fetal weight. Ultrasound Obstet
Gynecol. 2005;25(1):80-9.

5. Predanic M, Cho A, Ingrid F, Pelletieri J. Ultrasonographic estimation of fetal weight: acquiring accuracy in residency. J Ultrasound Med. 2002;21(5):495-500.

6. Gull I, Fait G, Har-Toov J, Kupferminc MJ, Lessing JB, Jaffa AJ, Wolman I. Prediction of fetal weight by ultrasound: the contribution of additional examiners. Ultrasound Obstet Gynecol. 2002;20(1):57-60.

7. Dudley NJ, Chapman E. The importance of quality management in fetal measurement. Ultrasound Obstet Gynecol. 2002;19(2):190-6.
8. Hadlock FP, Harrist RB, Sharman RS, Deter RL, Park SK. Estimation of fetal weight with the use of head, body, and femur measurements--a prospective study. Am J Obstet Gynecol. 1985;151(3):333-7.

9. Johnson RW, Toshach CE. Estimation of fetal weight using longitudinal mensuration. Am J Obstet Gynecol. 1954;68(3):891-6.

10. Nahum GG, Stanislaw H. Ultrasonographic prediction of term birth weight: how accurate is it? Am J Obstet Gynecol. 2003;188(2):566-74.

11. Anderson NG, Jolley IJ, Wells JE. Sonographic estimation of fetal weight: comparison of bias, precision and consistency using 12 different formulae. Ultrasound Obstet Gynecol. 2007;30(2):173-9.

12. Chien PF, Owen P, Khan KS. Validity of ultrasound estimation of fetal weight. Obstet Gynecol. 2000;95(6 Pt 1):856-60.

13. Siemer J, Egger N, Hart N, Meurer B, Müller $A$, Dathe 0 , et al. Fetal weight estimation by ultrasound: comparison of 11 different formulae and examiners with differing skill levels. Ultraschall Med. 2008;29(2):159-64.

14. Mirghani HM, Weerasinghe S, Ezimokhai M, Smith JR. Ultrasonic estimation of fetal weight at term: an evaluation of eight formulae. J Obstet Gynaecol Res. 2005;31(5):409-13.

Manuscrito recibido el 25 de mayo de 2009 y aceptado para publicación el 26 de junio de 2009.

Correspondencia:

Dr. José Ernesto Rojas Camayo

Jr. Canadá 319. San Martín de Porres

Lima 31, Perú

Correo-e: joserojas18@hotmail.com 